\title{
Des difficultés de compréhension du discours spécialisé en L2 par un public non expert. Le cas des étudiants polonophones de FLE face au discours académique
}

\author{
Some of the difficulties of a non-expert public \\ in understanding the second language specialised discourse. \\ The case of Polish students of the French philology \\ and the academic discourse
}

\author{
Anna Walicka \\ Université Adam Mickiewicz de Poznań \\ walickaa@amu.edu.pl
}

\begin{abstract}
The aim of the study reported in this article was to identify the knowledge or skills deficits which make Polish students of foreign languages fail to understand specialised discourse written in their second language (L2). The author analysed the translations proposed by French philology students for a fragment of a French academic text, particularly the translation errors consisting in source text's meaning transformation (i.e. misinterpretation, incorrect meaning, nonsense). The analysis allows to assume that these errors are the direct result of insufficient knowledge of the L2 in connection with wrong techniques of words' meaning verification.
\end{abstract}

Keywords: specialised discourse comprehension, translation errors, academic discourse

\section{INTRODUCTION}

Si les textes généraux font l'objet d'un travail pédagogique régulier à partir de la première classe de l'école primaire, force est de constater qu'il n'en est pas de même en ce qui concerne les textes spécialisés, comme si cette compétence se développait 
toute seule et qu'elle n'était utile et réservée que pour et dans la vie professionnelle. Or, la vie de tous les jours abonde en textes spécialisés : le discours juridique ou le discours économique sont omniprésents sous forme de contrats divers que signent les non experts (contrats de vente, de location, de prêt etc.) ou de documents qu'ils produisent (déclarations d'impôts par exemple). Certains arrivent à comprendre de tels textes, pour d'autres c'est un défi insurmontable. Il en va de même lorsque les textes spécialisés «adviennent » au cours d'une formation supérieure, qu'elle soit en L1 ou en L2.

Qu'est-ce qui décide de l'échec en compréhension des textes spécialisés par un public non expert? Peut-on arriver à définir une pertinence et/ou une profondeur «minimales» pour la compréhension d'un texte spécialisé par un public non expert? Voici les questions sur lesquelles nous voudrions nous pencher dans le présent article en limitant le champ de notre réflexion à la compréhension des textes spécialisés rédigés en L2. Défricher ce terrain importe d'un côté pour la didactique des langues étrangères (et plus particulièrement pour l'enseignement/apprentissage des discours spécialisés), et, de l'autre, pour la formation en traduction spécialisée. Définir les causes de l'incompréhension des textes spécialisés contribuera sans doute à l'amélioration de l'efficacité des outils existants ainsi qu'à l'élaboration de nouveaux outils pour l'enseignement/apprentissage des discours spécialisés et pour celui de la traduction spécialisée.

Pour nous rendre compte des difficultés que rencontre le public non expert lors de la compréhension des textes spécialisés rédigés en langue étrangère, nous avons décidé de procéder par l'analyse des traductions d'un fragment d'un texte académique français, réalisées par les étudiants polonophones de FLE en troisième année. L'étude que nous avons réalisée et qui est rapportée dans cette contribution, visait donc à identifier les sources de difficultés que présente pour les étudiants polonophones de FLE la lecture des textes académiques rédigés en français. Censés savoir comprendre ce type de discours, car obligés de rédiger leur licence en français, le savent-ils vraiment? Notre expérience de cinq ans de l'enseignement de la traduction du discours académique pour ce public atteste de nombreuses difficultés éprouvées lors de cette tâche. Les traductions réalisées par les étudiants contiennent de nombreuses fautes de sens (non-sens, sens contraire ou faux sens), témoins incontestables d'une incompréhension souvent très profonde du texte traduit. Les étudiants eux-mêmes avouent qu'ils ne comprennent pas le discours académique (voir également Orchowska, 2009, p. 186). Enquêtés sur les sources de leurs difficultés, ils évoquent en première position la maîtrise insuffisante du thème abordé dans le texte lu.

Leurs représentations correspondent-elles à la réalité ? Notre étude devait nous permettre justement de vérifier si c'est effectivement la maîtrise insuffisante du thème abordé dans les textes lus qui est responsable de l'incompréhension du discours académique par nos étudiants. Vu cet objectif, nous nous sommes concentrée dans nos analyses uniquement sur les fautes de sens présentes dans les traductions 
réalisées par les étudiants. Les résultats obtenus seront rapportés dans la deuxième partie de l'article tandis que sa première partie sera réservée principalement aux considérations d'ordre théorique portant sur les processus de compréhension et leur rôle déterminant pour l'opération traduisante, ceci pour démontrer que toute incompréhension du texte de départ laisse ses traces dans le texte d'arrivée. En deuxième lieu, nous préciserons dans la même partie deux notions clé qui se côtoient dans cette contribution, à savoir celle de texte de spécialité et celle de discours spécialisé. Etant donné divers usages terminologiques encore existants (surtout celui de « langue de spécialité »), une telle précision nous semble indispensable afin d'éviter toute confusion.

\section{CADRE THÉORIQUE}

\subsection{COMPRÉHENSION EN LECTURE}

Pour définir ce que veut dire « comprendre un texte », nous avons adopté la perspective cognitiviste dans laquelle les processus de compréhension de l'écrit sont examinés en termes de transformation de l'information.

Fournir une description de l'architecture cognitive et des fonctions mentales par lesquelles l'individu transforme l'information constitue un objectif commun des recherches menées par les représentants des disciplines différentes telles que la linguistique, les sciences de communication, l'intelligence artificielle, la psychologie cognitiviste, la philosophie du langage ou les neurosciences. L'étude des processus de compréhension fait ainsi partie des études plus larges sur la cognition humaine dans laquelle ces processus ont un rôle considérable.

Le fait que les hommes sont capables de connaître le monde dans lequel ils vivent, signifie qu'ils conservent des traces internes - ou représentations - de leurs interactions avec le monde environnant (Bronckart, 2001, p. 303). Chez l'humain, cette fonction «repose sur des unités représentatives délimitées (images mentales, sentiments, etc.) qui s'organisent en un système de pensée (opérations mentales) » (Bronckart, 2001, p. 303).

Etant donné que le langage est considéré dans cette perspective comme un système de représentations, et plus précisément « comme constitué de signes arbitraires, c'est-à-dire d'unités représentatives façonnées par l'échange social et radicalement indépendantes des propriétés des objets qu'elles désignent»(Bronckart, 2001, p. 305), la compréhension de l'écrit est conçue comme construction d'une représentation mentale du contenu lu dans un but déterminé (Karpińska-Szaj, 2005, p. 24).

$\mathrm{Du}$ fait que les structures et les fonctions des représentations sont déterminées par les fonctions de la mémoire, Le Ny distingue deux types de représentations : 
- les représentations occurrentes «conçues comme des événements mentaux, transitoires par nature, dont on doit inférer l'existence pour expliquer les comportements observables » (Karpińska-Szaj, 2005, p. 28) ;

- les représentations types « conçues comme des états mentaux, c'est-à-dire des structures mémorielles persistantes dont on doit inférer l'existence pour expliquer les représentations occurrentes. » (Karpińska-Szaj, 2005, p. 28).

«Dans un système de transformation de l'information, les représentations occurrentes décrivent l'état de l'information activée tout au long de la séquence de transformation effectuée dans la mémoire de travail, tandis que les représentations types décrivent un état initial de la mémoire, c'est-à-dire l'état de l'information mémorielle avant son activation lors du traitement de l'information nouvelle » (KarpińskaSzaj, 2005, p. 28).

Pour comprendre un texte, c'est-à-dire traiter des informations qu'il véhicule, l'individu doit donc construire ses représentations. Pour ce faire, il fait appel à des représentations types de la forme et des significations des mots qui forment un texte, à des représentations types de la structure du texte ainsi qu'à des connaissances sur le thème abordé dans le texte et sur la situation de communication.

\subsection{COMPRÉHENSION ET TRADUCTION}

C'est exactement ce que doit faire le traducteur pour traduire un texte : il doit le comprendre. Une forte corrélation entre la fidélité de la traduction par rapport au sens du texte de départ et la compréhension de ce texte n'est pas un axiome et a été confirmée de manière empirique (voir p. ex. Dancette, 1995). Il convient de noter que les mêmes recherches ont confirmé une forte corrélation également entre les compétences linguistiques et la fidélité des traductions (nous y reviendrons dans la suite de l'article).

Ainsi, la traduction révèle-t-elle des « indices » de compréhension permettant de juger de l'état de compréhension du texte traduit. Le résumé, le compte rendu ou la synthèse remplissent également le même rôle. Toutes ces productions, appelées aussi «métatextes » (Dancette, 1989), doivent être fidèles au texte source. La compréhension peut être vérifiée également par des réponses orales ou écrites à des questions portant sur un texte lu. Toutes ces techniques nous fournissent uniquement des indices de compréhension (ou d'incompréhension) et donnent uniquement un accès indirect aux processus de compréhension.

Parmi ces différentes techniques de recensement de données, c'est la traduction que nous avons retenue dans le cadre de notre recherche. Lire pour traduire n'est pas la même chose que lire pour extraire des informations ou pour comprendre l'idée générale du texte. Le traducteur ne peut sélectionner des informations à traduire mais il doit les restituer toutes fidèlement dans le texte d'arrivée. La traduction oblige ainsi à une compréhension très profonde du texte à traduire, tandis que la lecture 
réalisée pour résumer un texte ou pour en faire un compte rendu autorise une compréhension plus sélective par rapport à celle réalisée en traduction qui, de ce fait, permet de révéler des sources d'incompréhension de moindre "granularité ». Nous pensons notamment aux représentations types absentes ou erronées qu'il est plus facile de détecter dans les textes traduits que dans d'autres métatextes. En effet, les textes traduits reflètent les représentations types du sens de presque tous les mots du texte de départ, ce qui permet de cerner de manière très précise comme source d'incompréhension par exemple une représentation type erronée. Si notre observation portait uniquement sur le résumé, le compte rendu ou la synthèse, afin de détecter des représentations types absentes ou erronées, il serait indispensable de vérifier les significations possibles de chaque mot du texte à l'aide d'un questionnaire pointilleux.

Comment le texte traduit révèle-t-il des indices de compréhension ? Celui qui est fidèle au sens du texte de départ témoigne de la compréhension de ce texte par le traducteur. En revanche, les fautes de sens témoignent de l'incompréhension du texte de départ ou de ses fragments. Les fautes de sens, c'est-à-dire le non-sens, le contresens et le faux sens sont considérés par la plupart des théoriciens de la traduction comme des fautes de traduction qui trahissent les degrés différents d'incompréhension du texte de départ (voir Gouadec, 1974 ; Dancette, 1991 ; Plassard, 2007). Selon Gouadec, le non-sens est la faute la plus grave qui témoigne d'une incompréhension totale du texte ou d'une partie du texte à traduire. Il naît d'une méconnaissance des significations et de la syntaxe (grammaire).

L'incompréhension du texte de départ «ressort » ainsi dans le texte d'arrivée sous forme d'erreurs logiques, c'est-à-dire des passages, voire même des phrases entières présentant un non-sens, un faux sens ou un sens contraire par rapport au sens véhiculé par le texte de départ. Les fautes de sens sont donc des «indices» d'incompréhension. Les cas des traductions exactes sans qu'il y ait compréhension, sont des cas rares et impossibles lorsque le texte à traduire comporte des ambiguïtés lexicales, syntaxiques, morphologiques (Dancette 1991, p. 565).

\subsection{TEXTE DE SPÉCIALITÉ VS DISCOURS SPÉCIALISÉ}

Pour éclairer les usages terminologiques adoptés dans cette contribution, il convient de préciser la notion de texte de spécialité (dit aussi «spécialisé ») et celle de discours spécialisé ainsi que les rapports que ces notions entretiennent entre elles.

De manière délibérée nous avons évité dans cette contribution l'usage du terme « langue spécialisée » bien que le texte de spécialité ait été longtemps conçu comme «le produit de la langue de spécialité ». Cependant, ce qui portait longtemps le nom de «langue spécialisée » n'est pas une langue car ce n'est pas un système linguistique indépendant. Parler d'une langue semble ainsi pour le moins inexact. Une langue spécialisée (de spécialité) n'est pas non plus un sous-système, comme l'a 
défini Kocourek (1991) et à laquelle définition s'est opposé Lerat (Lerat, 1995, p. 18). Selon ce dernier, «la notion de langue spécialisée est pragmatique : c'est une langue naturelle considérée en tant que vecteur de connaissances spécialisées » (Lerat, 1995, p. 20). «Les langues spécialisées ne sont rien d'autre que des usages spécialisés des langues naturelles » (Lerat, 1997, p. 1).

Définir la langue spécialisée par un type d'usage de cette langue nous fait entrer immédiatement dans le domaine de la parole, ou plus particulièrement du discours. C'est pourquoi d'ailleurs l'appellation traditionnelle «langue spécialisée » se voit souvent remplacée par d'autres expressions jugées plus adéquates, telles que langues discursives, qui est une dénomination proposée par Grzmil-Tylutki (2014, p. 27), ou « discours spécialisé », expression fréquemment utilisée dans les travaux de Gajewska (2015) par exemple.

Le texte, qu'il soit spécialisé ou non, est souvent défini comme une production matérielle par opposition au discours qui n'est pas matériel, comme l'admettent de nombreux chercheurs (voir p. ex. Wilczyńska, 2012). En outre, comme le souligne Maingueneau, le texte est autonome par rapport à son contexte originel : «On a en effet tendance à parler de „texte” pour des productions verbales orales ou écrites qui sont structurées de manière à durer, à être répétées, à circuler loin de leur contexte originel » (Maingueneau, 2009, p. 34).

Par «texte de spécialité » nous comprenons ainsi toute production verbale écrite, structurée de manière à circuler loin de son contexte originel, émanant des spécialistes d'un domaine ou des personnes représentant une profession, une branche d'activité professionnelle, production ayant un contenu spécialisé.

Etant donné que «traduire c'est mettre un texte dans un discours différent » (Labocha, 2008, p. 79), l'opération traduisante oblige le traducteur à prendre en compte d'un côté le «discours de départ » que forment le texte de départ avec ses éléments paraverbaux et situationnels. De l'autre côté, dans la phase de production du texte d'arrivée, le traducteur doit prendre en compte également le «discours d'arrivée » qui renvoie alors à «un ensemble des règles de fonctionnement et d'interprétation d'énoncés, règles qui forment un modèle déterminé socialement, lié à un domaine (discours juridique, religieux) ou spécifique pour une communauté linguistique et culturelle donnée»(Labocha, 2008, p. 79). C'est en fonction des deux discours qu'il produit le texte d'arrivée.

\section{CADRE EXPÉRIMENTAL}

\subsection{DISPOSITIF EXPÉRIMENTAL ET SES MODALITÉS INSTITUTIONNELLES ET TECHNIQUES}

Cette partie de notre article fait état d'analyses élaborées à partir des traductions du fragment suivant : 
(...) Une autre contribution de Saussure à la linguistique est d'avoir défini la langue comme un système, ou encore comme une structure, terme qui sera privilégié par la suite et qui fera de la linguistique initiée par Saussure une linguistique structurale. La langue est un système en ce que le signe linguistique tire sa valeur de son rapport avec les autres signes, et non de lui-même : l'identité linguistique est relationnelle. Par exemple, le signifiant [lapin] tient son identité de sa différence avec d'autres signifiants tels que [lapon] ou [lopin]. De même, à l'écrit, la lettre «a » peut être formée de différentes façons. Elle reste un «a »dans les limites qui lui sont imposées par les différentes graphies

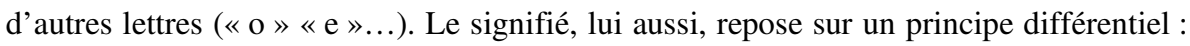
le sens de «cheval» se constitue par différence d'avec d'autres sens (ceux de «jument», «mulet», «étalon »...). La plus exacte caractéristique des signifiés, dit F. de Saussure, "est d'être ce que les autres ne sont pas». Le primat de la relation entre éléments sur les éléments eux-mêmes fait dire à Saussure que «dans la langue il n'y a que des différences ».

C'est un extrait de la contribution de Krieg à l'ouvrage « Le langage. Nature, histoire et usage » (2001). Ce court extrait, comptant 8 phrases à peine, a été traduit par un groupe de 20 personnes, étudiants en troisième année de philologie romane à l'Université Adam Mickiewicz à Poznań. Ces traductions ont été réalisées après neuf semaines d'exercices (tant collectifs qu'individuels) de traduction des textes académiques du domaine de la linguistique. Pendant la traduction, les étudiants disposaient de l'accès à l'internet et pouvaient consulter tout dictionnaire en papier. Il est à noter que cette dernière option n'a pas été choisie.

Il convient de préciser également que le programme de la formation en philologie romane introduit les exercices de traduction seulement en troisième année avec 60 heures de cours qui s'inscrivent dans l'ensemble des travaux pratiques (c'est-à-dire des cours consacrés uniquement à l'enseignement/apprentissage du français). Vu ce nombre d'heures très modeste ainsi que l'objectif global de tous les travaux pratiques, l'objectif du cours de traduction n'est pas de former des professionnels de traduction mais de développer la compétence de communication en L2 à travers les exercices de traduction. Afin d'adapter ce cours aux besoins des étudiants nous avons sélectionné comme son contenu uniquement des textes académiques du domaine de la linguistique. Leur traduction sert ainsi de méthode pour apprendre à comprendre les textes académiques.

\subsection{FAUTES DE SENS RECENSÉES : ANALYSE ET CATÉGORISATION}

La plupart des fautes de sens que nous avons recelées dans notre corpus, résultaient de l'attribution à un mot du texte de départ d'une signification qui n'était pas la sienne dans ce texte. Voici les lexèmes mal traduits (première colonne) avec leurs traductions erronées (deuxième colonne) accompagnées d'une explication de leur sens en français (troisième colonne) : 
Tableau 1. Fautes de sens

\begin{tabular}{|c|c|c|}
\hline $\begin{array}{l}\text { Lexèmes du texte de } \\
\text { départ }\end{array}$ & $\begin{array}{l}\text { Correspondants polonais choisis } \\
\text { par les étudiants }\end{array}$ & $\begin{array}{c}\text { Signification française } \\
\text { des correspondants polonais }\end{array}$ \\
\hline terme & $\begin{array}{l}\text { *pojęcie (2) } \\
\text { *forma słowa (1) }\end{array}$ & $\begin{array}{l}\text { concept } \\
\text { forme du mot }\end{array}$ \\
\hline faire & stanie się (1) & devenir \\
\hline linguistique structurale & *strukturalizm (1) & structuralisme \\
\hline en ce que & $\begin{array}{l}\text { non traduit (8) } \\
\text { jezzyk to system, *w którym (4) } \\
\text { poprzez który (1) }\end{array}$ & $\begin{array}{l}\text { dans lequel } \\
\text { à travers lequel }\end{array}$ \\
\hline tire sa valeur & \begin{tabular}{|l|} 
*czerpie korzyści (1) \\
*przyniesie korzyść (1) \\
\end{tabular} & $\begin{array}{l}\text { tire des profits } \\
\text { apportera un profit }\end{array}$ \\
\hline $\begin{array}{l}\text { le signe linguistique tire } \\
\text { sa valeur de son rapport } \\
\text { avec les autres signes, et } \\
\text { non de lui-même }\end{array}$ & $\begin{array}{l}\text { * w oparciu o inne znaki, a nie o siebie } \\
\text { samego (1) } \\
\text { *ze związków z innymi znakami, ale nie } \\
\text { z samym sobą (4) }\end{array}$ & de son rapport avec lui-même \\
\hline défini & posiadanie (1) & posséder \\
\hline privilégié & *zastrzeżony (1) & réservé \\
\hline par la suite & $\begin{array}{l}\text { *w następstwie (2) } \\
\text { *od tego czasu (2) } \\
\text { *odtąd (1) }\end{array}$ & depuis, désormais \\
\hline identité & *świadomość (1) & conscience \\
\hline identité linguistique & $\begin{array}{l}\text { tożsamość *językoznawcza (1) } \\
\text { tożsamość *lingwistyczna (2) } \\
\text { *językoznawstwo (1) }\end{array}$ & qui se rapporte à la linguistique \\
\hline relationnelle & $\begin{array}{l}\text { *zgodna z rozsądkiem (1) } \\
\text { *racjonalna (2) }\end{array}$ & rationnelle \\
\hline sa différence avec & różnica *między (1) & entre \\
\hline a l'écrit & $\begin{array}{l}\text { *piśmiennictwo (2) } \\
\text { *język pisany }\end{array}$ & $\begin{array}{l}\text { littérature } \\
\text { langue écrite }\end{array}$ \\
\hline dans les limites & w *ograniczeniach (2) & dans les contraintes \\
\hline signifié & $\begin{array}{l}\text { *forma }(1) \\
\text { *rzecz }(1)\end{array}$ & $\begin{array}{l}\text { forme } \\
\text { chose }\end{array}$ \\
\hline signifiant & $\begin{array}{l}\text { *signifiant słowa (2) } \\
\text { *znaczony (1) } \\
\text { *znaczenie (2) słowa } \\
\text { *znak językowy } \\
\end{array}$ & $\begin{array}{l}\text { signifiant du mot } \\
\text { signifié } \\
\text { sens du mot } \\
\text { signe linguistique } \\
\end{array}$ \\
\hline repose & $\begin{array}{l}\text { *polega (1) } \\
\text { *działa (1) } \\
\end{array}$ & $\begin{array}{l}\text { consiste } \\
\text { fonctionne }\end{array}$ \\
\hline se constitue & *jest uznawane (1) & est admis/considéré \\
\hline primat & $\begin{array}{l}\text { *sedno (1) } \\
\text { *naczelna relacja (1) } \\
\text { *najwyższy autorytet (1) }\end{array}$ & $\begin{array}{l}\text { l'essentiel } \\
\text { relation qui prime } \\
\text { l'autorité supérieure }\end{array}$ \\
\hline primat de ... sur ... & $\begin{array}{l}\text { nadrzędność ... *i ... (1) } \\
\text { pierwszeństwo ... *wśród ... (1) } \\
\text { priorytetowość/prymat ... *na ... (3) }\end{array}$ & $\begin{array}{l}\text { et } \\
\text { parmi }\end{array}$ \\
\hline
\end{tabular}




\begin{tabular}{|c|c|c|}
\hline $\begin{array}{l}\text { Lexèmes du texte de } \\
\text { départ }\end{array}$ & $\begin{array}{l}\text { Correspondants polonais choisis } \\
\text { par les étudiants }\end{array}$ & $\begin{array}{c}\text { Signification française } \\
\text { des correspondants polonais }\end{array}$ \\
\hline & $\begin{array}{l}\text { pierwszeństwo relacji elementów *między } \\
\text { elementami (1) } \\
\text { prymat związku pomiędzy elementami } \\
\text { *w elementach (1) } \\
\text { prymat związku między różnymi elemen- } \\
\text { tami *i elementami samymi w sobie (1) } \\
\text { prymat relacji pomiędzy elementami } \\
\text { *a elementami (1) } \\
\text { *prymatem relacji elementów jest stwier- } \\
\text { dzenie (1) }\end{array}$ & $\begin{array}{l}\text { entre (au lieu de «sur ») } \\
\text { dans } \\
\text { préposition non-traduite avec } \\
\text { l'omission d'une partie de la } \\
\text { phrase }\end{array}$ \\
\hline caractéristique & *charakterystyka (15) & description \\
\hline différences & *sprzeczności (1) & contradictions \\
\hline fait dire à Saussure & $\begin{array}{l}\text { *według de Saussure'a (1) } \\
\text { *jak powiedział de Saussure (6) } \\
\text { de Saussure *powiedział } \\
\text { przekonuje do słów de Saussure'a (1) } \\
\text { prymat relacji (...) *mówi/*przekazuje de } \\
\text { Saussure'owi (1) }\end{array}$ & $\begin{array}{l}\text { selon Saussure } \\
\text { comme Saussure a dit } \\
\text { Saussure a dit } \\
\text { convainc aux mots de Saussure } \\
\text { le primat dit à Saussure }\end{array}$ \\
\hline
\end{tabular}

TOTAL N : 20

Quels facteurs ont influencé le choix d'un correspondant inexact ? L'analyse du tableau permet de constater que les confusions des significations des noms ou des adjectifs dues à une typographie voisine, à la polysémie ou à l'interférence sont des problèmes rares. En effet, seul un mot, à savoir l'adjectif « relationnelle », a été mal décodé et confondu avec « rationnelle ». Il ne s'agissait pas non plus de problèmes dus à la polysémie, car également un seul adjectif «linguistique », renvoyant et à la langue (językowy), et à la linguistique (językoznawczy), était une source d'erreur en traduction due à la polysémie. L'interférence des formes linguistiques a provoqué également une seule faute : le substantif «caractéristique » a été traduit par charakterystyka (« description »). Un petit nombre de fautes résultait de la méconnaissance de la signification du correspondant polonais : deux personnes ont notamment rendu «l'écrit » par piśmiennictwo et l'expression «dans les limites » par w ograniczeniach (au lieu de $w$ granicach).

Aucune confusion n'explique pourtant pourquoi les autres mots recensés dans le tableau se voient attribuer des significations qui ne sont pas les leurs et ne peuvent pas être même considérées comme leurs significations potentielles. De nombreuses fautes commises par exemple dans la traduction du substantif «primat » témoignent d'une simple méconnaissance de la signification de ce mot (rendu en polonais par «*l'essentiel » ou «*l'autorité »). Du même problème témoignent également des omissions très fréquentes, concernant par exemple l'expression «en ce que »: 8 étudiants sur 20 ont omis cette expression dans leurs traductions dont le sens 
n'était plus identique à celui du texte de départ. Bien que les significations des substantifs « signifié », « signifiant», « valeur », « terme » et « concept » aient été connues des étudiants grâce aux textes traduits auparavant, notre analyse a démontré qu'elles n'ont tout simplement pas été mémorisées, ou qu'elles ont été mémorisées incorrectement.

La maîtrise insuffisante de la syntaxe française constitue une autre cause de fautes de sens assez fréquentes. La construction «faire faire qqch à qqn » s'est avérée inconnue de la moitié des étudiants. Par conséquent, le fragment suivant de la dernière phrase « $(. .$.$) fait dire à Saussure que (. .$.$) » est rendu dans leurs traductions$ d'une manière complètement erronée sans qu'il reste dans le texte polonais la moindre trace de la structure «faire dire ». Dans les traductions proposées par les étudiants, nous retrouvons ainsi un faux sens (p.ex. «selon Saussure», "Saussure a dit ») ou un non-sens (« le primat dit à Saussure »).

De nombreuses fautes de sens apparaissent dans la traduction des prépositions dont l'importance pour la construction du sens semble souvent négligée par les étudiants. Ainsi, dans ce fragment provenant de la deuxième phrase du texte : « le signe linguistique tire sa valeur de son rapport avec les autres signes, et non de luimême », 5 personnes ne remarquent point qu'il ne s'agit pas du rapport du signe linguistique «avec » lui-même, et rendent la préposition «de » (qui dans le texte de départ signifie l'origine, le retour à soi) par la préposition «avec». Dans la version polonaise, le sens de ce passage devient alors complètement faux. La faute est d'autant plus sérieuse que cette phrase exprime une idée très importante qui est ensuite reprise dans la dernière phrase du fragment, à savoir « Le primat de la relation entre éléments sur les éléments eux-mêmes fait dire à Saussure que 'dans la langue il n'y a que des différences' », phrase qui constitue une sorte de conclusion du fragment entier.

Les prépositions se sont d'ailleurs avérées des indicateurs très précieux de l'incompréhension du sens de l'idée exprimée dans les deux phrases. Nous l'avons remarqué surtout grâce aux difficultés que les étudiants ont rencontrées lors de la traduction de la dernière phrase : 10 personnes sur 20 étaient incapables de traduire correctement les prépositions «sur» et «entre » présentes dans cette phrase. Etant donné qu'elle exprime l'idée principale du fragment entier, sa traduction erronée témoigne de l'incompréhension du fragment entier.

Nous supposons qu'en grande partie c'est la méconnaissance de la signification du substantif «primat» qui en était responsable. En effet, les prépositions « entre » et «sur » ne sont souvent pas traduites correctement ou ne sont pas traduites du tout, comme si les étudiants ne connaissaient que la forme des correspondants polonais du mot «primat» sans savoir vraiment ce que signifie ce mot. Les formulations polonaises proposées par les étudiants, telles que relacja elementów *między elementami («relation des éléments *entre les éléments ») ou prymat zwiazku pomiędzy elemen- 
tami $*$ w elementach («le primat de la relation entre les éléments *dans les éléments ») sont des non-sens complets.

Cet exemple à lui seul confirme que s'il suffit parfois de remplacer un substantif français par son correspondant polonais pour obtenir une traduction correcte (sans comprendre le texte de départ), il ne peut en être de même pour les prépositions. Elles «servent à relier des termes pour les intégrer dans une construction plus vaste » (Riegel, Pellat, Rioul, 2009, p. 639), ce qui veut dire que l'on ne peut généralement trouver leurs correspondants « hors » discours. Leur rôle est pourtant crucial pour la compréhension car «elles contribuent à l'établissement de relations sémantiques entre les termes qu'elles relient » (Riegel, Pellat, Rioul, 2009, p. 639).

Pour conclure, dans la majorité des cas que nous avons recensés dans le tableau ci-dessus, l'attribution à un mot d'une signification qui n'est pas la sienne témoigne d'une simple méconnaissance des significations de mots particuliers, d'expressions ou de structures syntaxiques. Cette méconnaissance empêche évidemment la compréhension du sens des phrases et du texte entier.

\section{CONCLUSION}

L'étude que nous avons réalisée a démontré que l'incompréhension du discours académique par les étudiants polonophones de FLE n'était pas due directement et uniquement à la maîtrise insuffisante des thèmes abordés des les textes lus, comme ils le pensaient, mais elle résultait surtout de la méconnaissance des significations des mots particuliers. Une grande majorité de ces mots n'appartenaient pas à la terminologie spécialisée, ce qui aurait pu expliquer éventuellement certaines difficultés de compréhension.

Il s'est avéré ainsi que c'est la maîtrise insuffisante de la langue de départ qui a directement bloqué la compréhension des textes spécialisés rédigés en français par les étudiants de FLE. Dépourvus des connaissances du domaine, ils ne pouvaient plus compenser leurs déficits linguistiques par ces connaissances, comme ils le font afin de comprendre le sens des textes généraux. Les représentations des étudiants n'étaient donc pas complètement fausses : s'ils avaient mieux connu le domaine qu'est la linguistique, ils auraient sans doute profité de leurs connaissances du domaine là où leurs connaissances linguistiques ne suffisaient pas pour comprendre le sens du texte lu. Notre étude a ainsi confirmé également le lien entre les connaissances linguistiques et la fidélité de la traduction, lien déjà démontré dans d'autres travaux (Dancette, 1995).

Certaines fautes de sens que nous avons décelées permettent de supposer en outre que certains étudiants ne savent pas exploiter toutes les ressources internet pour vérifier la signification des mots inconnus. Autorisés à explorer les ressources électroniques dans leurs recherches terminologiques, les étudiants ont fini par renon- 
cer complètement à tout dictionnaire en papier et ils exploraient uniquement des ressources accessibles sur internet parmi lesquelles le dictionnaire le plus souvent utilisé a été celui de PONS. Il semble très significatif que ce dictionnaire ne contienne pas le correspondant polonais du substantif «primat » qui a posé tant de problèmes de traduction. Les déficits portant sur les stratégies de la recherche terminologique sur internet mériteraient ainsi d'être vérifiés et examinés de près dans le cadre d'une étude spécifique que nous pensons entreprendre. Améliorer ces stratégies semble une voie prometteuse vers une bonne maittrise de la L2 qui, comme notre étude l'a démontré, conditionne de manière inéluctable la compréhension du discours spécialisé. 


\section{BIBLIOGRAPHIE}

Bronckart, J.-P. (2001). Langage et représentations. In J.-F. Dortier (réd.), Le langage. Nature, histoire et usage (pp. 303-308). Auxerre : Éditions Sciences Humaines.

Dancette, J. (1989). La faute de sens en traduction. TTR, 2(2), 83-102. doi : 10.7202/037048a

Dancette, J. (1991). Les opérations de compréhension préalables à la production de métatextes : recherche d'une typologie. Revue belge de philologie et d'histoire, 69, 563-575.

Dancette, J. (1995). Parcours de traduction. Étude expérimentale du processus de compréhension. Lille : Presses Universitaires de Lille.

Gajewska, E. (2015). Badania nad dyskursami specjalistycznymi a praktyka pedagogiczna. In M. Sowa, M. Mocarz-Kleindienst, U. Czyżewska (réd.), Nauczanie języków obcych na potrzeby rynku pracy (pp. 39-52). Lublin : Wydawnictwo KUL.

Grzmil-Tylutki, H. (2014). Langues spécialisées ou langues discursives - question terminologique ou choix épistémologique? Roczniki humanistyczne, LXII (8), 27-47. URL : http://czasopisma.tnkul. $\mathrm{pl} /$ index.php/rh/article/view/1358/1474

Gouadec, D. (1974). Comprendre et traduire. Paris : Bordas.

Karpińska-Szaj, K. (2005). Pédagogie de la lecture en langue étrangère. Défis rééducatifs. Poznań : Wydawnictwo Naukowe UAM.

Krieg, A. (2001). Ferdinand de Saussure, le père fondateur de la linguistique moderne. In J.-F. Dortier (réd.), Le langage. Nature, histoire et usage (pp. 21-24). Auxerre : Éditions Sciences Humaines.

Kocourek, R. (1991). Textes et termes. META, 36 (1), 71-76. URL: https://www.erudit.org/en/jour nals/meta/1991-v36-n1-n1/003330ar/

Labocha, J. (2008). Tekst, wypowiedź, dyskurs w procesie komunikacji językowej. Kraków : Wydawnictwo Uniwersytetu Jagiellońskiego.

Lerat, P. (1995). Les langues spécialisées. Paris : PUF.

Lerat, P. (1997). Approches linguistiques des langues spécialisées. ASp, 15-18, 1-10. doi : $10.4000 /$ asp. 2926

Maingueneau, D. (2007[2009]). Analyser les textes de communication. Paris : Armand Colin.

Orchowska, I. (2009). Rozwijanie kompetencji dyskursywnej studentów KJO w zakresie dyskursu naukowego: wyzwania językowe, dyskursywne i interkulturowe. In J. Nijakowska (réd.), Język poznanie - zachowanie. Perspektywy $i$ wyzwania $w$ studiach nad przyswajaniem języka obcego (pp. 173-189). Łódź : Wydawnictwo Uniwersytetu Łódzkiego.

Plassard, F. (2007). Lire pour traduire. Paris : Les Presses de la Sorbonne Nouvelle.

Riegel, M., Pellat, J.-Ch., Rioul, R. (1994[2009]). Grammaire méthodique du français. Paris : Presses Universitaires de France.

Wilczyńska, W. (2013). Dyskurs - brakujące ogniwo nierozerwalności kultury i języka, czyli o integracji w kształceniu neofilologów. Lingwistyka Stosowana, 8, 133-152.

Wilczyńska, W. (2012). Czy pojęcie dyskursu jest przydatne w glottodydaktyce? Neofilolog, 38 (1), $7-25$. 